TITLE:

\title{
Irradiation effects on solid surfaces by water cluster ion beams
}

$\operatorname{AUTHOR}(S)$ :

Ryuto, Hiromichi; Tada, Keiji; Takaoka, Gikan H.

\section{CITATION:}

Ryuto, Hiromichi ...[et al]. Irradiation effects on solid surfaces by water cluster ion beams. Vacuum 2009, 84(5): 501-504

ISSUE DATE:

2009-12-10

URL:

http://hdl.handle.net/2433/89518

\section{RIGHT:}

c 2009 Elsevier Ltd. All rights reserved.; この論文は出版社版でありませ ん。引用の際には出版社版をご確認ご利用ください。; This is not the published version. Please cite only the published version. 


\title{
Irradiation Effects on Solid Surfaces by Water Cluster Ion Beams
}

\author{
Hiromichi Ryuto ${ }^{*, 1}$, Keiji Tada, and Gikan H. Takaoka \\ Photonics and Electronics Science and Engineering Center, Kyoto University \\ Nishikyo, Kyoto 615-8510, Japan
}

\begin{abstract}
The interaction between a water cluster ion beam and the surface of a silicon substrate was investigated. The sputtering yield of silicon by a water cluster ion beam was approximately ten times larger than that by an argon monomer ion beam. Xray photoelectron spectroscopy was used to analyze the silicon surface irradiated with a water cluster ion beam. The analysis revealed that the surface was oxidized, and the oxidation was saturated approximately at the dose of $1 \times 10^{14}$ ions $/ \mathrm{cm}^{2}$. The number of disordered atoms measured by the Rutherford backscattering also supported the result.
\end{abstract}

Key words: cluster ion beam, sputtering, oxidation, surface modification PACS: 36.40.-c, 68.49.Sf, 68.55.Ln

\footnotetext{
* Corresponding author Email address: ryuto@kuee.kyoto-u.ac.jp (Hiromichi Ryuto).

1 Formerly, H. Akiyoshi
} 


\section{Introduction}

In the information society, the demand on the further application of water is growing within the development of the next generation semiconductor devices. For example, the quest for up-to-date cleaning methods that cross the limit of conventional wet processes has intensified. On the other hand, the ion beam techniques such as an ion beam etching and implantation are common and powerful techniques in the semiconductor manufacturing. Recently, another type of ion beam technique appeared, a cluster ion beam technique. Clusters are aggregates of molecules with sizes ranging from dimers to thousands of monomers. The cluster ion beam method realizes an irradiation of electromagnetically controlled ion beams that are more than one hundred times heavier than the heaviest atomic ion beam, a ${ }^{238} \mathrm{U}$ beam. The unique properties of the interaction between cluster ions and solid materials, such as a high sputtering yield and surface smoothing effect [1], are mainly attributable to a high-density energy deposition and a low-energy irradiation effect. In this article, an approach to a brand-new utilization of water, a water cluster ion beam technique is described.

\section{Experimental Procedure}

Figure 1 shows a schematic view of the water cluster ion beam apparatus. A series of four vacuum chambers form the apparatus. In the leftmost vacuum chamber in the Fig. 1, a stainless steel container filled with purified water is heated by a cable heater attached to the outer wall of the container to vaporize water. A typical quantity of water is $250 \mathrm{~mL}$ for approximately $2 \mathrm{~h}$ irradiation. 
The temperature of the container is kept at the temperature that corresponds to the required vapor pressure $\left(p_{0}\right)$ of water. For example, the temperature corresponding to the typical vapor pressure of $3 \mathrm{~atm}$ is $134^{\circ} \mathrm{C}$. The temperature is maintained at approximately within $\pm 1^{\circ} \mathrm{C}$, which corresponds to the vapor pressure variation of $\pm 9 \mathrm{kPa}$. The vaporized water is emitted to the vacuum chamber through a Laval nozzle. The vapor is cooled by adiabatic expansion, and clusters are formed [2]. The core portion of the stream is selected by a skimmer, and transported to the next vacuum chamber. The remaining vapor is evacuated using a roots pump. The vacuum pressures of the other three chambers are lower than the chamber containing the liquid container, and they are evacuated using diffusion pumps. The next vacuum chamber is used for differential pumping, and the neutral clusters are transported to the third vacuum chamber. In the third chamber, the neutral clusters are ionized by the electrons emitted from a loop of tungsten filament and accelerated by the voltage applied between the filament and an anode mesh. The typical voltage and current of the ionization electrons are $200 \mathrm{~V}$ and $200 \mathrm{~mA}$, respectively. The ionized cluster ions are extracted by the extraction voltage applied between the anode and an extraction electrode. The monomers and the small-sized clusters are eliminated using the retarding voltage $\left(V_{r}\right)$ applied to the retarding electrode. It was reported that the velocity of the nozzle flow is highly uniform typically within $10 \%[2,3]$, so the kinetic energy of a cluster is proportional to the cluster size (the number of molecules that compose a cluster). The kinetic energy of water clusters produced at the vapor pressure of $3 \mathrm{~atm}$ is $0.14 \mathrm{eV} /$ molecule [4]. The size-separated cluster ions are accelerated by the acceleration voltage $\left(V_{a}\right)$ applied between the retarding electrode and an acceleration electrode. The typical acceleration voltage was from 3 to 9 $\mathrm{kV}$. The transverse divergence of the cluster ion beam is suppressed using an 
Einzel lens, and the beam is transported to the sample placed in the rightmost vacuum chamber in Fig. 1. The sample and a Faraday cup were attached to a linear motion feedthrough, and one of them was placed at the peak position of the horizontal beam intensity distribution. A voltage of approximately $-300 \mathrm{~V}$ is applied to the electron suppressor of the Faraday cup. The vertical position of the beam is fine-tuned by an electrostatic deflector. A cluster size distribution produced with a vapor pressure of $3 \mathrm{~atm}$ measured by the time-of-flight method has a peak approximately at the cluster size of 2500 [5]. The time variation of the beam intensity was approximately $10 \%$. The samples were $\mathrm{Si}(100)$ or $\mathrm{SiO}_{2}$ substrates. The sputtered depths of the samples were measured using a step profiler. The composite elements of the irradiated surfaces were measured by the X-ray photoelectron spectroscopy (XPS). The numbers of disordered atoms were measured by the Rutherford backscattering method of $\alpha$ particles at $2 \mathrm{MeV}$.

\section{Results and Discussion}

Figure 2 shows the acceleration voltage dependences of the sputtered depths in silicon and silicon dioxide substrates irradiated by water cluster ion beams. The dose was $1 \times 10^{16}$ ions $/ \mathrm{cm}^{2}$. The sputtered depths both in silicon and silicon dioxide substrates increased with increase in the acceleration voltage. The sputtered depths of the silicon dioxide substrates were larger than those of the silicon substrates. The sputtering yields of the silicon and silicon dioxide substrates irradiated by $9 \mathrm{keV}$ water cluster ion beams were $18 \mathrm{Si}$ atoms/ion and $15 \mathrm{SiO}_{2}$ molecules/ion assuming a non-selective sputtering and densities of 2.3 and $2.2 \mathrm{~g} / \mathrm{cm}^{3}$, respectively. Moreover, the sputtering yield of the silicon 
substrate irradiated by a water cluster ion beam at $9 \mathrm{keV}$ was approximately 10 times larger than that irradiated by an argon monomer ion beam at the same incident energy.

Figure 3 shows Si 2p XPS spectra of the silicon surfaces irradiated by water cluster ion beams with doses from $1 \times 10^{13}$ to $1 \times 10^{16}$ ions $/ \mathrm{cm}^{2}$. Two peaks appeared on the Si $2 p$ spectrum due to chemical shifts. The peaks at $99.5 \mathrm{eV}$ and $103.3 \mathrm{eV}$ are assigned to the silicon atoms in the silicon crystal and the silicon atoms of the silicon dioxide, respectively [6]. The size of the peak attributable to the silicon dioxide increased and the size of the peak attributable to the silicon crystal decreased with the increase in the dose of the water cluster ion beam irradiation. The difference between the spectra of $1 \times 10^{13}$ ions $/ \mathrm{cm}^{2}$ and $1 \times 10^{14}$ ions $/ \mathrm{cm}^{2}$ were distinct, but the difference among the spectra of $1 \times 10^{14}$ ions $/ \mathrm{cm}^{2}, 1 \times 10^{15}$ ions $/ \mathrm{cm}^{2}$, and $1 \times 10^{16}$ ions $/ \mathrm{cm}^{2}$ were limited. It may be explained as the silicon atoms near the surface of the silicon crystal were oxidized to form silicon dioxide, and the oxidation was soon saturated approximately at the dose of $1 \times 10^{14}$ ions $/ \mathrm{cm}^{2}$.

Figures 4 show (a) Si 2p and (b) O 1s XPS spectra in the silicon substrates whose depth profiles were measured using an argon sputtering. In Fig. 4 (a), the size of the peak attributable to the silicon dioxide decreased and the peak attributable to the silicon crystal increased with increase in the sputtered depth. Similarly, in Fig. 4 (b), the size of the O 1s peak decreased with increase in the sputtered depth. Moreover, the peak from the silicon dioxide in the Si $2 \mathrm{p}$ spectrum and the $\mathrm{O}$ 1s peak almost disappeared when the depth was 10.6 nm. Therefore, the thickness of the oxidized layer produced by the irradiation of the water cluster ion beam may be approximately $10 \mathrm{~nm}$. On the other hand, the peak from the silicon dioxide and the $\mathrm{O}$ 1s peak shifted to the lower 
binding energy side with the increase in the depth, which may indicate the variation of the chemical composition from silicon dioxide. It can be said that the sputtering yield of silicon is possibly suppressed by the intense oxidation effect.

Figure 5 shows acceleration voltage dependences of the number of disordered atoms in silicon substrates caused by the irradiation of water cluster and argon monomer ion beams. A number of disordered atoms is recognized as the measure of lattice disorders in a silicon crystal [7]. In the case of the irradiation of a water cluster ion beam, the measured number of disordered atoms possibly includes the contributions from both the recoiling effect in atomic collisions and the oxidation effect. The acceleration voltage dependence of the numbers of disordered atoms in silicon substrates irradiated by water cluster ion beams had a rather common tendency with those irradiated by argon monomer ion beams. Both of the numbers of disordered atoms increased with the increase in the acceleration voltage. However, the numbers of disordered atoms in silicon substrates irradiated by water cluster ion beams were systematically smaller than those irradiated by argon monomer ion beams.

Figure 6 shows dose dependences of the number of disordered atoms in silicon substrates caused by the irradiation of water cluster and argon monomer ion beams. The numbers of disordered atoms induced by the argon monomer ion beam irradiation monotonically increased with the increase in the dose. Apart from the argon monomer ion beam case, the numbers of disordered atoms induced by the water cluster ion beam saturated approximately at the dose of $1 \times 10^{14}$ ions $/ \mathrm{cm}^{2}$. This result may support the result of the XPS measurement, which showed the saturation approximately at the dose of $1 \times 10^{14}$ ions $/ \mathrm{cm}^{2}$. 


\section{Conclusion}

The sputtering yield of silicon substrate by a water cluster ion beam was approximately ten times higher than that caused by an argon monomer ion beam. The irradiation of a water cluster ion beam caused an oxidation on the surface of the silicon substrate, which saturated approximately at the dose of $1 \times 10^{14}$ ions $/ \mathrm{cm}^{2}$.

\section{Acknowledgements}

A part of this work was conducted in Kyoto-Advanced Nanotechnology Network, supported by "Nanotechnology Network" of the Ministry of Education, Culture, Sports, Science and Technology (MEXT), Japan. The RBS measurements were performed at the Quantum Science and Engineering Center, Kyoto University.

\section{References}

[1] I. Yamada, Nucl. Instrum. Methods. B 148 (1999) 1.

[2] O. F. Hagena, W. Obert, J. Chem. Phys. 56 (1972) 1793.

[3] O. F. Hagena, K. Varma, Rev. Sci. Instr. 39 (1968) 47.

[4] O. F. Hagena, in: P. P. Wegener (Ed.), Molecular Beams and Low Density Gas Dynamics, Dekker, New York, 1974, pp. 93-181.

[5] G. H. Takaoka, H. Noguchi, K. Nakayama, M. Kawashita, Rev. Sci. Instrum. 77 (2006) 03B509. 
[6] J. F. Moulder, W. F. Stickle, P. E. Sobol, K. D. Bomben, in: J. Chastain, R. C. King, Jr. (Ed.), Handbook of X-ray Photoelectron Spectroscopy, Physical Electronics, Eden Prairie, 1995.

[7] W.-K. Chu, J. W. Mayer, M.-A. Nicolet, Backscattering Spectrometry, Academic Press, New York, 1978. 
Figure captions:

Fig. 1: Schematic view of water cluster ion beam apparatus.

Fig. 2: Acceleration voltage dependence of sputtered depths of $\mathrm{Si}$ and $\mathrm{SiO}_{2}$ substrates irradiated by water cluster ion beams.

Fig. 3: Si $2 p$ XPS spectra of silicon substrates irradiated by water cluster ion beams with doses from $1 \times 10^{13}$ to $1 \times 10^{16}$ ions $/ \mathrm{cm}^{2}$.

Fig. 4: (a) Si 2p and (b) O 1s XPS spectra of silicon substrate irradiated by water cluster ion beam. The measurements were performed at the depths from 0 to $10.6 \mathrm{~nm}$ by using Ar sputtering.

Fig. 5: Acceleration voltage dependences of number of disordered atoms in silicon substrates irradiated by water cluster and Ar monomer ion beams.

Fig. 6: Dose dependences of number of disordered atoms in silicon substrates irradiated by water cluster and Ar monomer ion beams at $6 \mathrm{keV}$. 


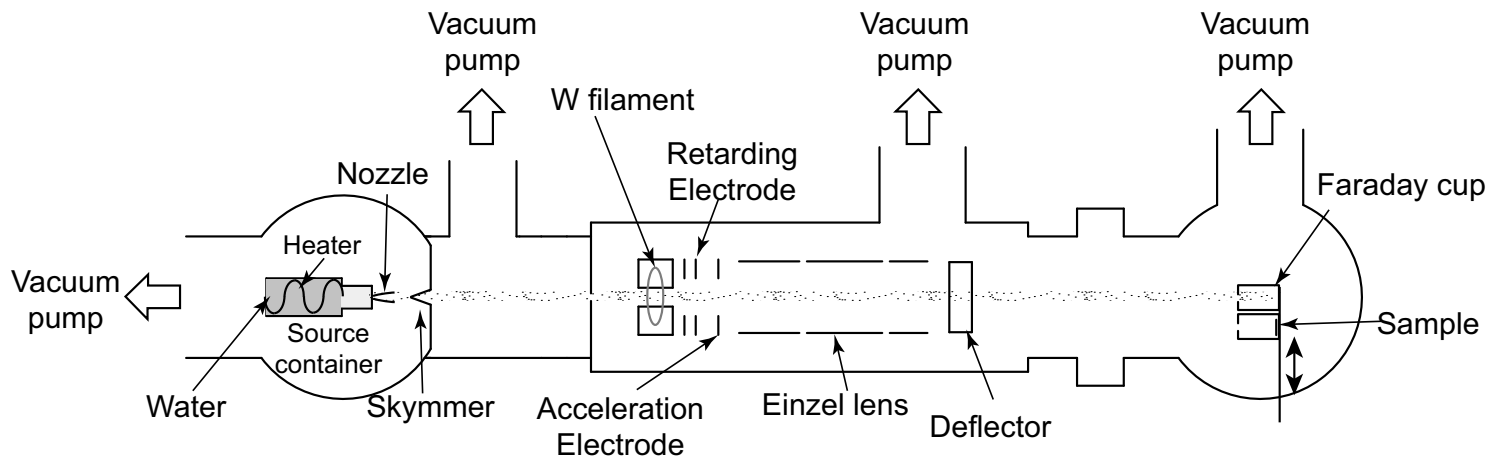

Fig. 1. 


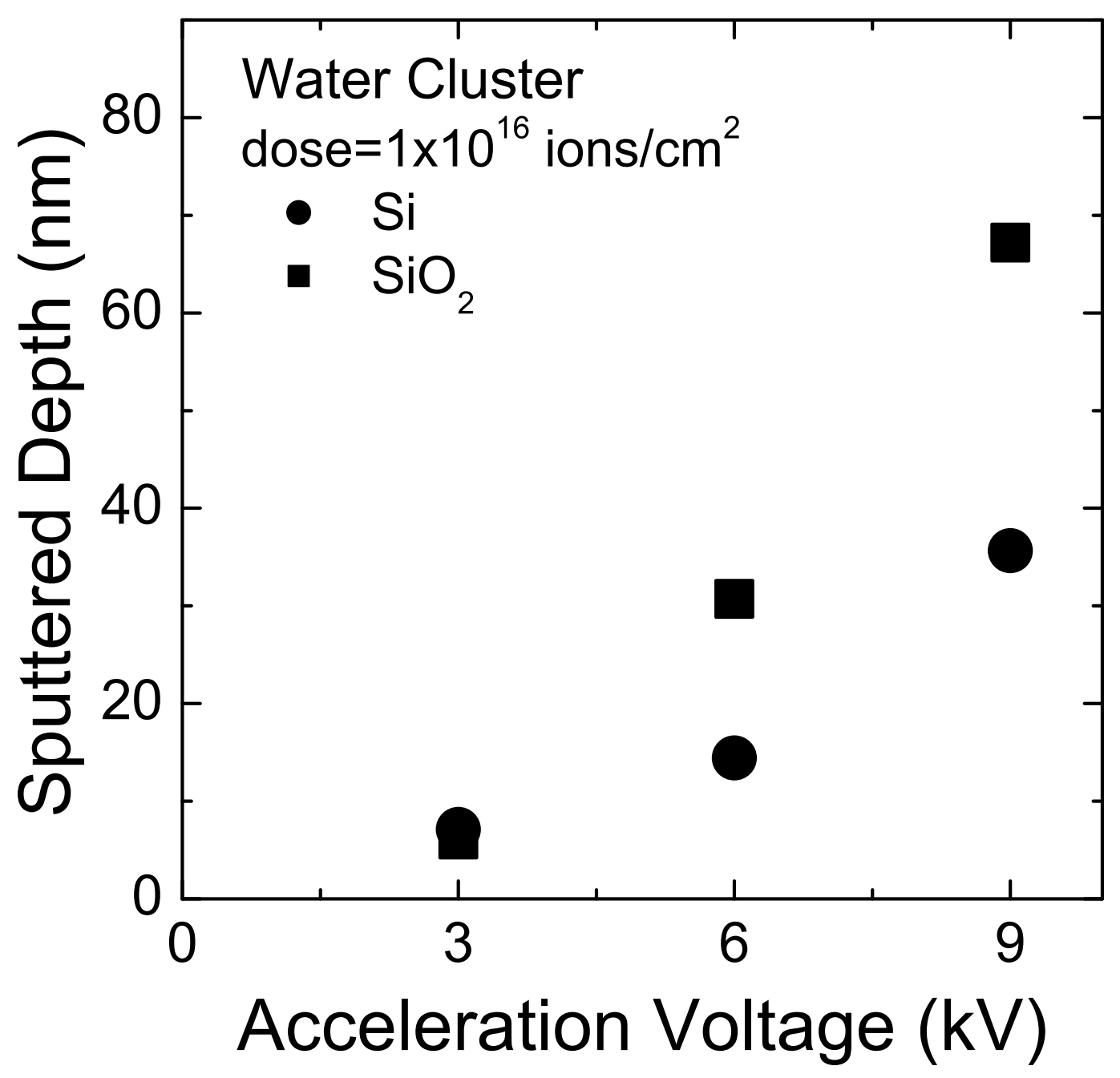

Fig. 2. 


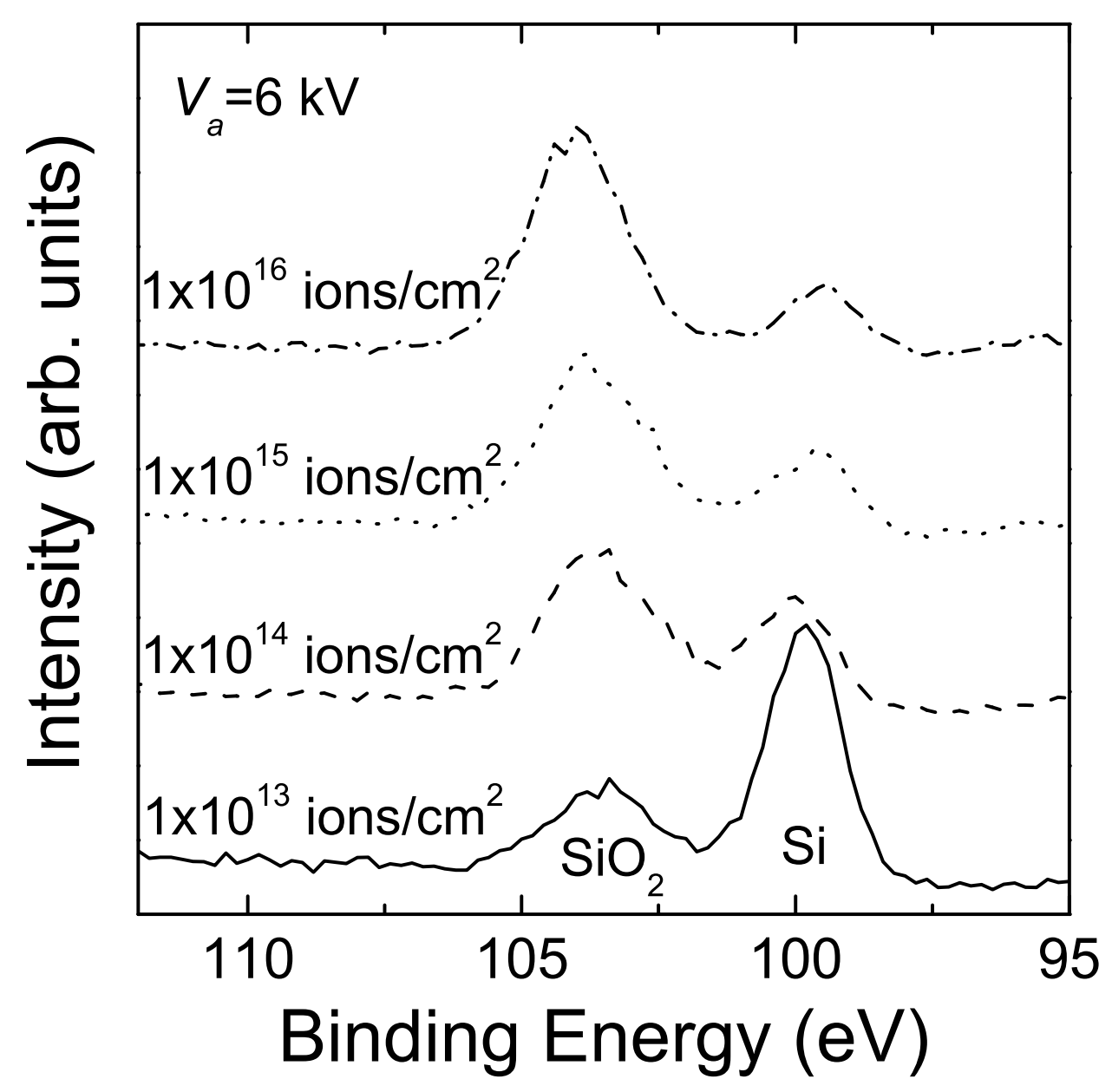

Fig. 3. 


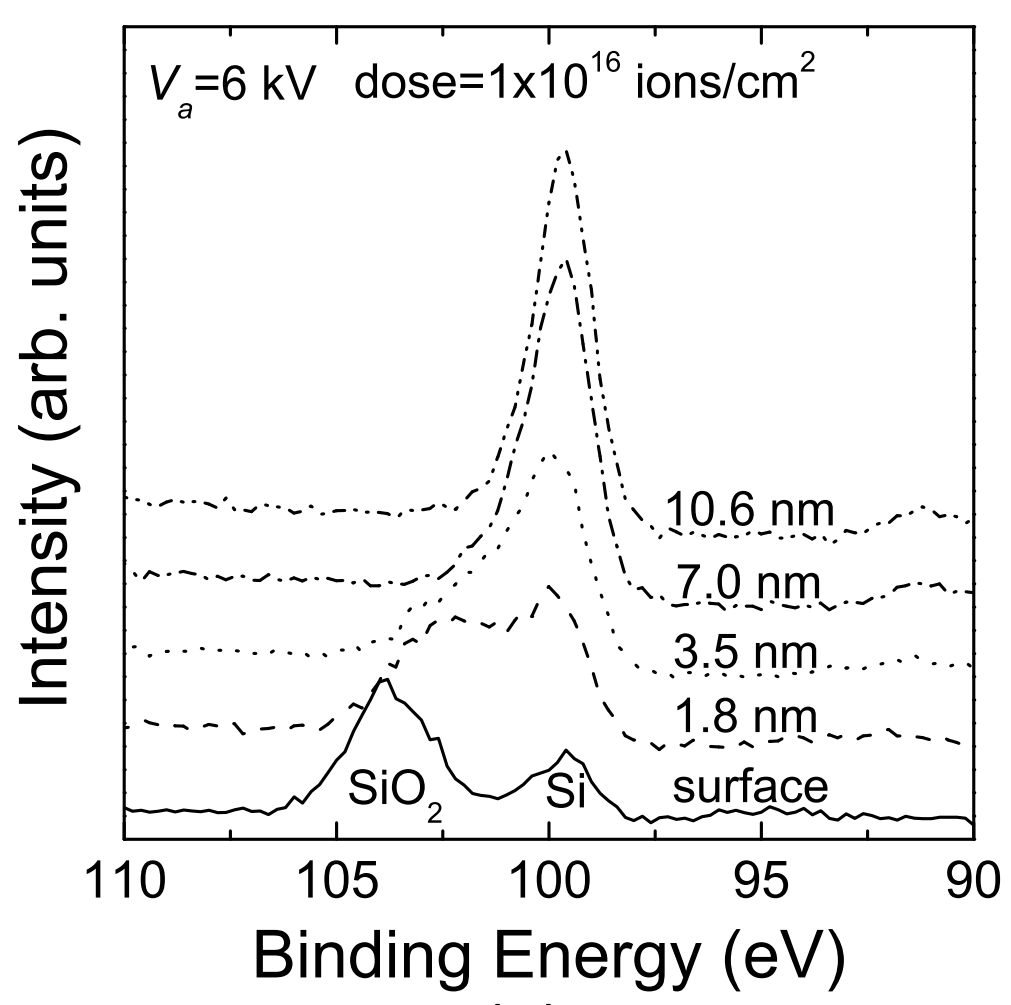

(a)

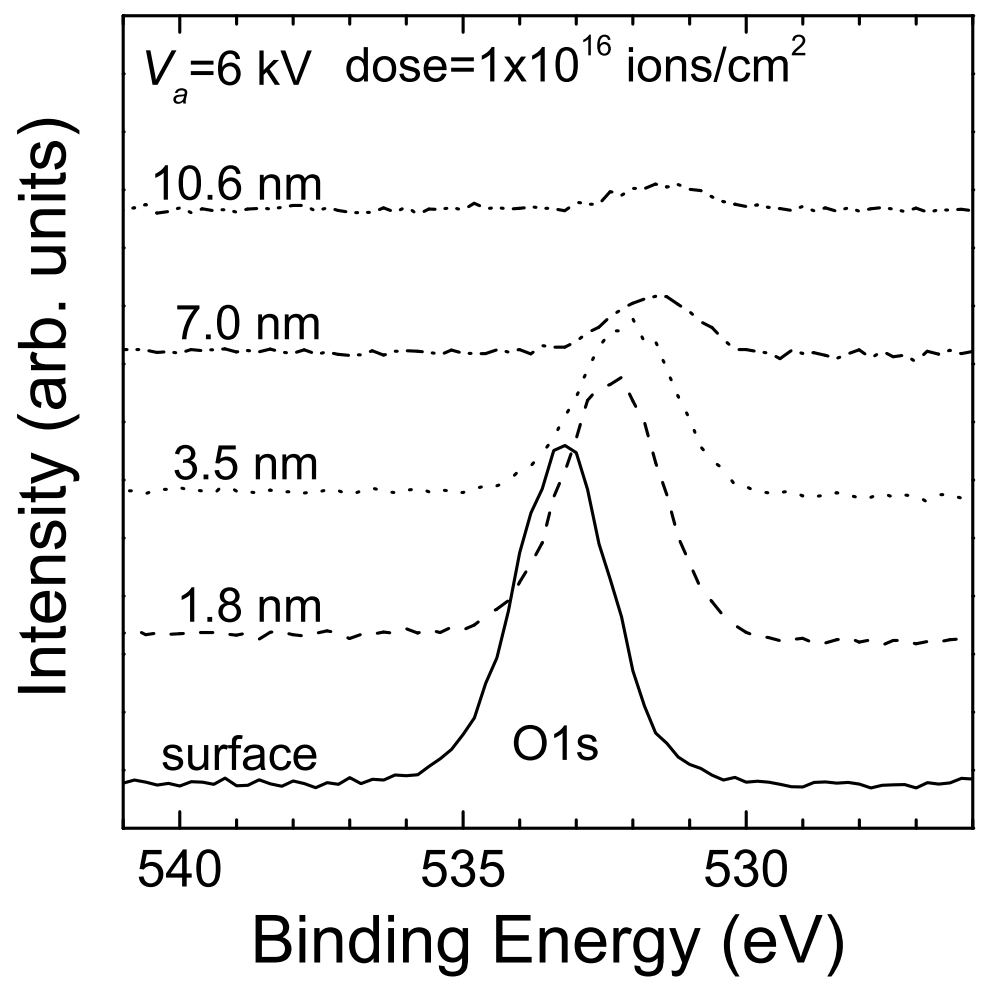

(b)

Fig. 4. 


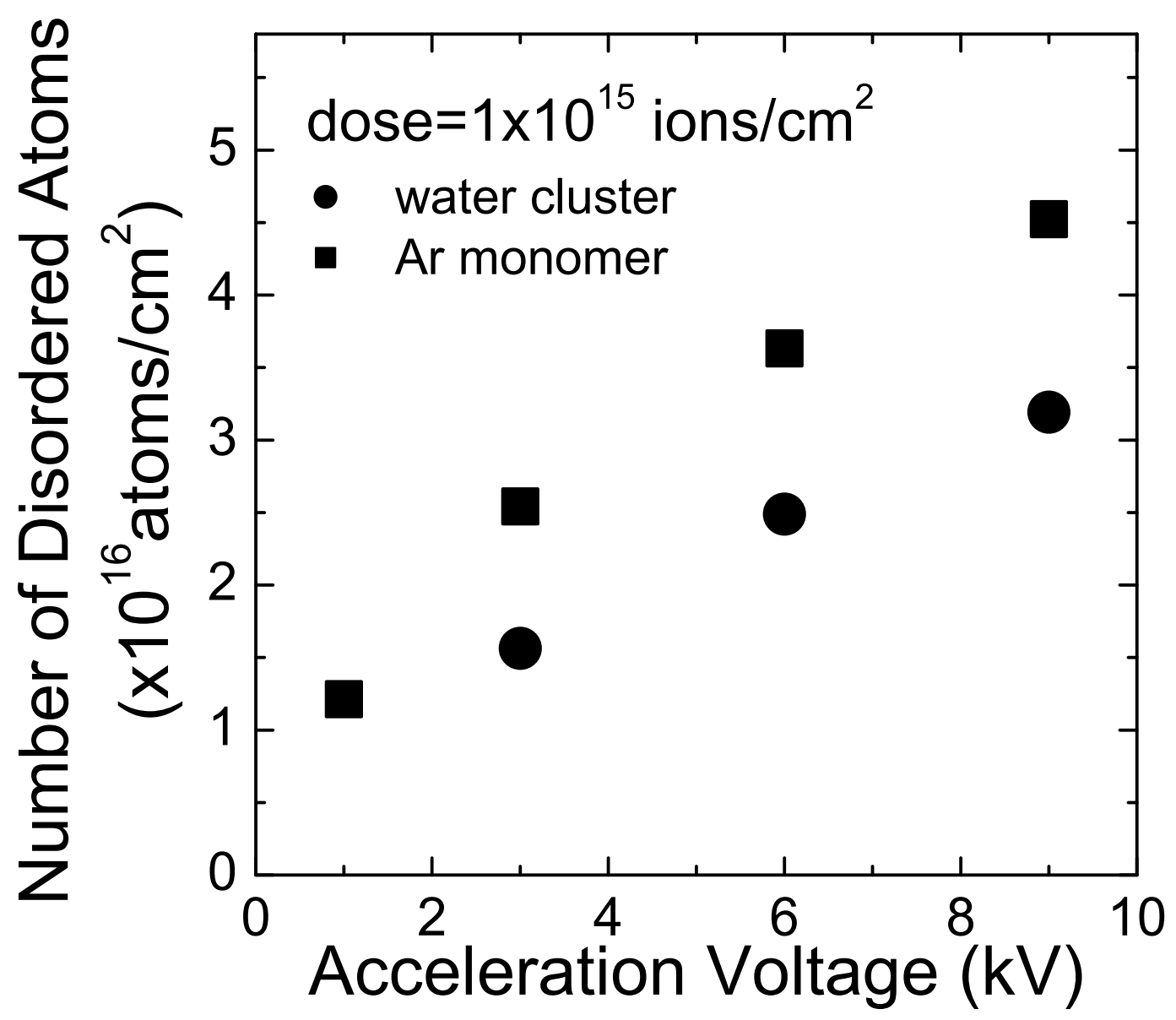

Fig. 5. 


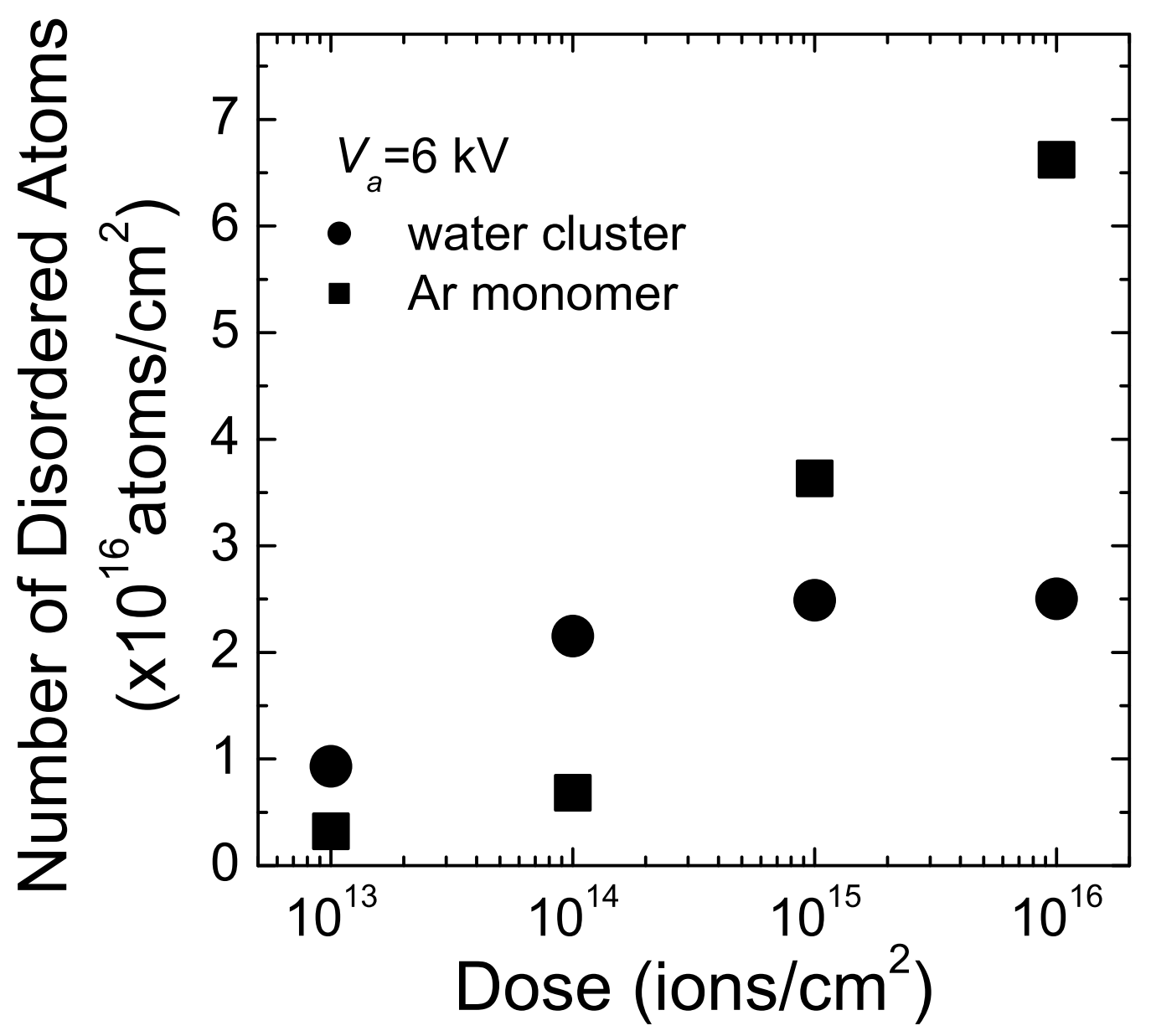

Fig. 6. 